Immunogenetics 15: 357-364, 1982

\title{
Liver Tissue Graft Rejection in Murine Major Histocompatibility Complex Mutants
}

\author{
Jane S. Schultz ${ }^{1 *}$, Roberta DeMott-Friberg ${ }^{2}$, and Theodore F. Beals ${ }^{3}$ \\ 1 VA Medical Center and University of Michigan, Department of Human Genetics, Ann Arbor, \\ Michigan \\ 2 VA Medical Center, Ann Arbor, Michigan \\ ${ }^{3}$ VA Medical Center and University of Michigan, Department of Pathology, Ann Arbor, Michigan
}

\begin{abstract}
Liver tissue grafts between seven $H-2$ mutants and their parental strains have been studied. Each of these mutants was originally identified by reciprocal mutant - parental strain skin graft rejection. However, liver grafts among mutants and parental standard strains are not uniformly rejected. Liver graft rejection also fails to correlate with mutant - parental stimulation in CML and MLC. In addition, the immune reaction pattern of female mutant animals against grafts of male liver differs from the reaction pattern found in parental standard strains. Several explanations for the differences between immune response to liver and skin grafts are proposed, including different $\mathrm{T}$ cell subsets involved in recognition, availability of antigenic sites to immunocompetent cells, and structural differences between mutant and parental H-2 antigens.
\end{abstract}

\section{Introduction}

Twenty-seven $H-2$ mutants have been identified by one or another of three laboratories (Nairn et al. 1980). The inbred lines that developed from these mutants have further facilitated genetic analyses of the $\mathrm{H}-2$ complex, as well as the assignment of immunological reactivities to its regions. These mutants were identified by unexpected skin graft rejection when mice were screened by the skin graft method of Bailey and Usama (1960) or some modification thereof. In addition to skin graft reactivity, mutant strains exhibit histogenic differences from their parental strains as detected by a variety of tests (reviewed by Klein 1978, Kohn et al. 1978, Egorov 1974, Ben-Nun et al. 1980, Egorov et al. 1979, McKenzie et al. 1979, Wu et al. 1980, Streilein and Klein 1980, Bennett et al. 1980, Melief et al. 1980, Davis

\footnotetext{
* Present Address: VA Medical Center-151, 2215 Fuller Road, Ann Arbor, Michigan, 48105 Abbreviations used in this paper: bm1, 2, 3, 4, 14; dm1; $\mathrm{fm} 2=$ mutants of strains $\mathrm{C} 57 \mathrm{BL} / 6, \mathrm{~B} 10 . \mathrm{D} 2$ and $\mathrm{B} 10 . \mathrm{M}$ respectively; $\mathrm{B} 6=\mathrm{C} 57 \mathrm{BL} / 6$
} 
et al. 1980). These tests, which include cell mediated lymphocytotoxicity (CML), mixed lymphocyte culture (MLC), graft vs. host reactivity (GVHR), susceptibility to autoimmune thyroiditis, T-cell function, hybrid resistance, tolerance induction and heart graft rejection, do not all show equivalent mutant-parental strain differences. Serological differences between parents and mutants have been difficult to demonstrate in most cases.

Recently the class 1 molecules of several of these mutants (Brown and Nathenson 1977, Brown et al. 1978, Ewenstein et al. 1980, and Nisizawa et al. 1981) have been subjected to structural analyses. These studies have shown mutantparental differences in the amino acid sequences of the H-2K, D and/or L structural proteins varying from one to several amino acids (reviewed by Nairn et al. 1980). We have studied seven $\mathrm{H}-2$ mutant strains in the liver slice to kidney bed grafting system (Schultz et al. 1976). These mutants differ from their standard strains of origin as demonstrated by the ability to acutely reject skin grafts and by their vigorous CML and MLC reactivity. However, serological reactivity and quantitative reactions in CML and MLC are not identical in all standard strain-mutant, and mutant-mutant combinations. The published values for these immunological parameters are summarized in Table 1. We have previously demonstrated (Schultz et al. 1976, Schultz et al. 1978) that genetic control of liver to kidney bed graft survival in inbred strains and congenic lines is not identical to the control of skin graft survival. It is, therefore, of interest to determine whether liver graft survival among mutants and between mutant and standard strains is in agreement with skin graft survival data. We are also interested in the predictive value of in vitro cellular immune assays such as the CML and MLC and serological typing for liver graft survival. The correlation of changes in the biochemical structure of the H-2K, H-2D, and/or H-2L gene products with the observed survival of either skin or liver to kidney grafts is also of importance. The results of the following studies have supplied answers to some of these questions.

\section{Materials and Methods}

\footnotetext{
Mutant and parental strains were obtained from the following sources: B6.C-H-2 ${ }^{b m 1}(b m 1), \mathrm{B} 6-H-2^{b m 3}$ $(b m 3)$, and B10.D2-H-2 ${ }^{d m 1}(d m 1)$ were bred in our laboratory from breeders originally supplied by Dr. Jan Klein of the Max Planck Institute, Tübingen, Federal Republic of Germany. Breeders for the remaining mutant strains [B6.C-H-2 $2^{b m 2}(b m 2)$, B6.C-H-2 ${ }^{b m 4}(b m 4), \mathrm{B} 6 \mathrm{C}-\mathrm{H}-2^{b m 14}(b m 14)$, and B10.M-H-2 $\left.{ }^{f m 2}(f m 2)\right]$ were obtained from Dr. Donald Bailey of The Jackson Laboratory. The strains B10.M and B10.D2 were bred in our animal colony at the VA Medical Center in Ann Arbor, Michigan. Strains C57BL/10 (B10), B10.D2, and C57BL/6 (B6) were obtained from The Jackson Laboratory and the National Cancer Institute.

The liver-to-kidney bed grafting system employed has been described in detail previously (Schultz et al. 1976). In brief, a small piece of liver taken from an anesthesized donor is placed on the etherized recipient's left kidney, which has been prepared by removing a shallow subcapsular slice. The recipient's abdominal incision is sutured and the mouse returned to the animal colony. At predetermined times, the recipient mice are killed by cervical dislocation. Grafted kidneys are removed, fixed, trimmed, embedded, sectioned, and stained. Numerous sections of each grafted kidney are screened to locate the central area of the graft. Three slides containing sections from the most central areas of the graft are then evaluated.

Histological scores are based on a previously reported evaluation system (Schultz et al. 1978). This system uses "P" to identify a proliferating graft with more than four layers of hepatocytes with or without cellular immunological activity, "S" to identify a syngeneic-like graft with 3-4 well organized layers of
} 
Table 1. Previously reported data on immunological parameters of selected mutants

\begin{tabular}{|c|c|c|c|c|c|c|c|}
\hline $\begin{array}{l}\text { Donor or } \\
\text { stimulator } \\
\text { locus of } \\
\text { mutation }\end{array}$ & $\begin{array}{l}\text { Recipient or } \\
\text { responder } \\
\text { locus of } \\
\text { mutation }\end{array}$ & $\begin{array}{l}\text { Mean Survival } \\
\text { Time (MST) of } \\
\text { skin grafts (days) }\end{array}$ & $\begin{array}{l}\text { Serological } \\
\text { differences }\end{array}$ & $\mathrm{CML}$ & $\begin{array}{l}\text { Activity } \\
\text { index } \\
\text { MLC }\end{array}$ & $\begin{array}{l}\text { Heart } \\
\text { graft } \\
\text { rejection } \\
\%\end{array}$ & Source \\
\hline$b m 1(\mathrm{H}-2 \mathrm{~K})$ & B6 & 16.5 & $\pm *$ & + & 5.9 & NT & \\
\hline B6 & $b m 1(\mathrm{H}-2 \mathrm{~K})$ & 15.1 & \pm & + & 5.8 & 58 ( 6 weeks $)$ & ศ \\
\hline$b m 2(\mathrm{H}-2 \mathrm{~K})$ & B6 & $t^{\neq}$ & NT & NT & NT & NT & \\
\hline B6 & $b m 2(\mathrm{H}-2 \mathrm{~K})$ & + & NT & NT & NT & NT & \\
\hline$b m 3(\mathrm{H}-2 \mathrm{~K})$ & B6 & 21.8 &,$+ \pm^{\dagger}$ & + & 7.7 & 70 (6 weeks) & $\square$ \\
\hline B6 & $b m 3(\mathrm{H}-2 \mathrm{~K})$ & 18.5 &,+ \pm & + & 8.7 & 50 (4 weeks) & $\square$ \\
\hline$b m 4(\mathrm{H}-2 \mathrm{~K})$ & B6 & + & + & + & + & NT & \\
\hline $\mathrm{B} 6$ & $b m 4(\mathrm{H}-2 \mathrm{~K})$ & + & + & + & + & NT & \\
\hline$b m 14(\mathrm{H}-2 \mathrm{D})$ & B6 & + & NT & + & NT & NT & \\
\hline B6 & $b m 14(\mathrm{H}-2 \mathrm{D})$ & + & NT & + & NT & NT & \\
\hline$d m 1(\mathrm{H}-2 \mathrm{D}, \mathrm{H}-2 \mathrm{~L})$ & B10.D2 & 16.2 & + & + & 2.5 & 80 (5 weeks) & 0 \\
\hline B10.D2 & $d m 1(\mathrm{H}-2 \mathrm{~K}, \mathrm{H}-2 \mathrm{~L})$ & 17.0 & + & + & 9.2 & 70 ( 4 weeks $)$ & 0 \\
\hline$f m 2(\mathrm{H}-2 \mathrm{D})$ & B10.M & + & + & + & + & NT & \\
\hline B10.M & $f m 2(\mathrm{H}-2 \mathrm{D})$ & + & + & + & + & NT & \\
\hline$b m 1(\mathrm{H}-2 \mathrm{~K})$ & $b m 3(\mathrm{H}-2 \mathrm{~K})$ & 14.0 &,+ \pm & + & NT & NT & $\triangle$ \\
\hline$b m 3(\mathrm{H}-2 \mathrm{~K})$ & $b m l(\mathrm{H}-2 \mathrm{~K})$ & 15.0 &,+ \pm & + & $\mathrm{NT}$ & NT & $\triangle$ \\
\hline
\end{tabular}

* $\pm=$ quantitative difference only

$\dagger+, \pm=$ only one weak antigenic difference and quantitative difference

$\neq+=$ rejected - M.S.T. not available

" by cytotoxic test using antisera against standard H-2 specificities- bm 1 and bm4 grafts fail to elicit antibodies in parental strain nor do parental strain grafts elicit antibodies in bm1 and bm4-dm1 grafts elicit antibodies in B10.D2. The reciprocal is also true.

$\$$ General sources: Klein 1978 and Kohn et al. 1978

I Davis et al. 1980

${ }^{\circ}$ Egorov 1974

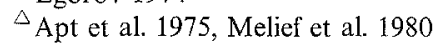

$\square$ Klein 1976

hepatocytes with little or no cellular immunological activity, "C-1" to identify a healthy graft with a few lymphocytes concentrated or in small nodules at the graft-bed interface, "CC-2" to identify a graft containing less healthy, poorly organized hepatocytes with a considerable number of lymphocytes scattered throughout the graft, "C-3" to identify a graft containing degenerating scattered hepatocytes, copious lymphocytic infiltrate and fibrovascular tissue, "C-4" to identify a graft with lymphocytes present in large nodules and with only a few scattered hepatocytes in a young scar, "C-5" to identify a graft consisting of fibrovascular tissue with some collagen (young scar), a few lymphocytes present and no hepatocytes in evidence, and "C-6" to identify a graft with only a matured (contracted) scar from which most lymphocytes have receded. The appearance of a graft identified as " $\mathrm{C}-6$ " is indistinguishable from the appearance of an acutely rejected graft such as seen across an H-2 barrier (DeMott-Friberg et al. 1979).

\section{Results}

Our results for liver graft to kidney bed survival, summarized in Figure 1, show control syngeneic male to male liver grafts surviving at $70 \mathrm{~d}$ after grafting in three $\mathrm{H}$ $2^{b}$ mutant strains ( $\left.b m 1, b m 3, b m 14\right)$. Male to male grafts in the $d m 1$ strain, although uniformly surviving, contain some immunologically competent cells. 
Male to female grafts in mutant strains $b m 1$ and $b m 3$ are rejected by $b m 1$ females $56 \mathrm{~d}$ after grafting and by $b m 3$ females $70 \mathrm{~d}$ after grafting. Male to female grafts in $b m 14$ and $d m 1$ survive at $70 \mathrm{~d}$ after grafting although immunological activity is clearly present in the grafts. Some male to female grafts in the B6 standard strain survive at $105 \mathrm{~d}$ after grafting; all grafts are not rejected until $140 \mathrm{~d}$ after grafting.

Reciprocal grafts between mutant and standard strains show a variable survival pattern. Some grafts from B6 males to $b m 1$ males, B6 to $b m 3$ males and $b m 3$ males to B6 males are in chronic immunologic difficulty but few are rejected by $70 \mathrm{~d}$ after grafting. However, $b m 1$ to $\mathrm{B} 6$ and $b m 4$ to $\mathrm{B} 6$ male to male liver grafts are uniformly rejected by $70 \mathrm{~d}$. Only one of seven $b m 2$ to $\mathrm{B} 6$ grafts is surviving at $70 \mathrm{~d}$. Grafts from the $\mathrm{H}-2 \mathrm{D}^{\mathrm{b}}$ mutant, $b m 14$, to its standard parental, $\mathrm{B} 6$, and the reciprocal of these grafts survive with only very minor immunological activity.

Reciprocal grafts differ radically in the $d m 1$ to B10.D2 combination. While all $d m 1$ to B10.D2 grafts are rejected by $35 \mathrm{~d}$ after grafting, B10.D2 to $d m 1$ grafts still survive at $140 \mathrm{~d}$ after grafting. Grafts survive in the $\mathrm{fm} 2$ to B $10 . \mathrm{M}$ combination and its reciprocal.

A variety of reactions occur in grafts among mutant strains and these, too, differ in reciprocal combinations. Grafts from $b m 14$ males to $b m 1$ males survive with some immunologic activity at $70 \mathrm{~d}$ after grafting, while $b m 1$ to $b m 14$ grafts are rejected completely in seven of eight animals sampled at $70 \mathrm{~d}$. There is moderate immunological activity in $b m 14$ to $b m 3$ grafts at $70 \mathrm{~d}$ after grafting. The reciprocal combination is also in immunologic difficulty, but grafts survive in all but one animal. Only five of 18 grafts between $b m l$ and $b m 3$ animals survive at $70 \mathrm{~d}$ after grafting.

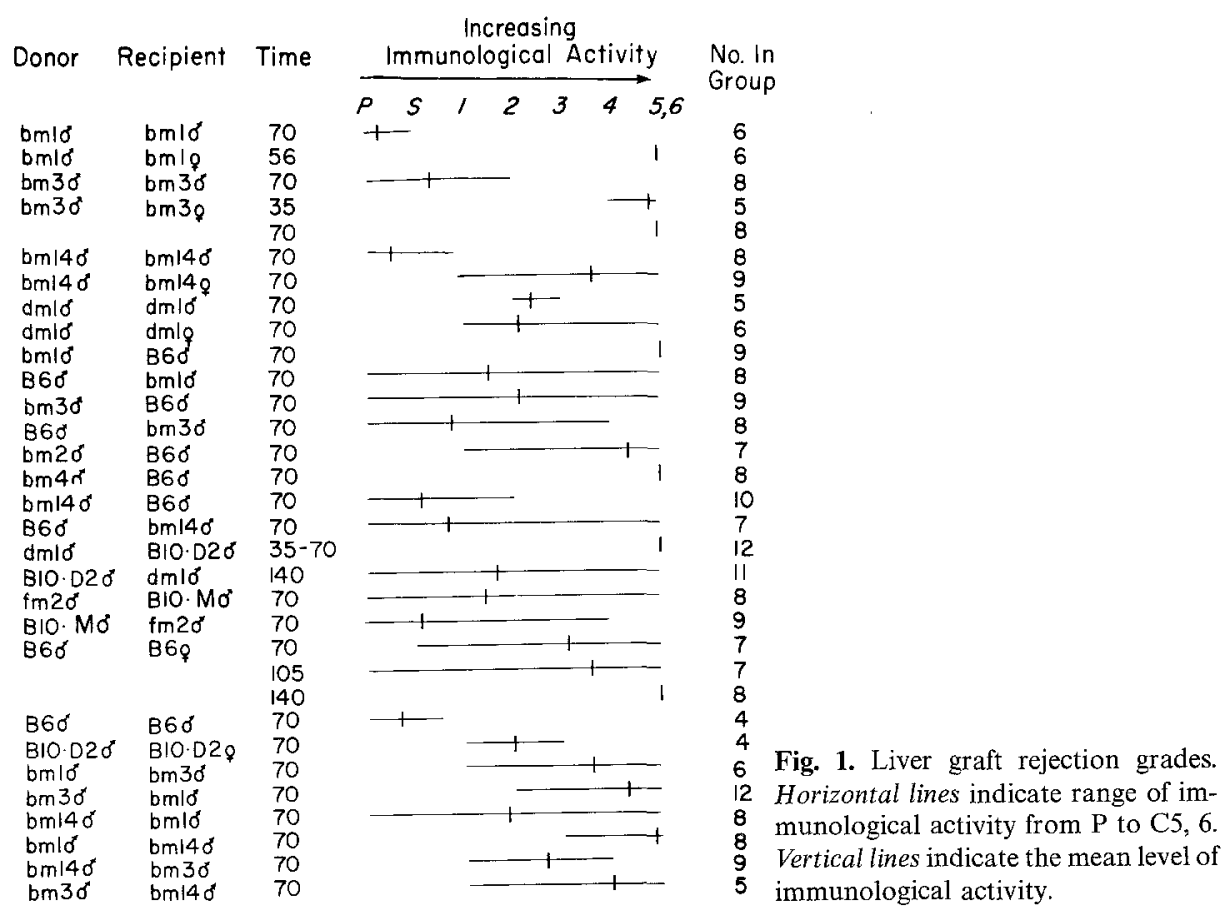




\section{Discussion}

Although all the mutants included in this study were originally identified by their ability to acutely reject skin grafts from their standard strains of origin (see Table 1), different results are obtained with liver tissue to kidney bed grafts. Skin grafts are rejected more easily than liver grafts (Table 1 and Fig. 1). One possible reason for this difference between skin graft and liver graft survival in mutants is the region in which the mutation is located. All of the $H-2^{b}$ mutants studied are $H-2 \mathrm{~K}$ region mutations except for $b m 14$ which has been assigned to the $H-2 D$ region. Immunological activity in liver grafts in the $b m 14$ to B6 mutant-parental strain combination is extremely low compared with activity in $b m 4$ to $\mathrm{B} 6$ and $b m 1$ to $\mathrm{B} 6$ combinations. However, $b m 3$ to B 6 grafts survive at $70 \mathrm{~d}$ after grafting. Therefore, $\mathrm{H}-2$ regional locale of mutations does not entirely explain the difference in behavior of skin and liver grafts in $\mathrm{H}-2^{b}$ mutants.

Biochemical analysis has revealed that the $b m 3$ mutant has amino acid alterations at positions 77 and 89 in $\mathrm{CNBr}$ fragment $\mathrm{Ib}$ of the $\mathrm{H}-2 \mathrm{~K}^{\mathrm{b}}$ peptide (Ewenstein et al. 1980). Two amino acid residues, 155 and 156 in CNBr fragment Ia of the $\mathrm{H}-2 \mathrm{~K}^{\mathrm{b}}$ peptide, are altered in the $b m 1$ mutant. Mutant $b m 4$ also has an altered Ia CNBr fragment (cited in Nairn et al. 1980).

Mutants $b m 1$ and $b m 4$ perform similarly in the liver graft system, in that grafts from them to the B 6 standard strain are rejected by $70 \mathrm{~d}$ after grafting. Grafts from the $b m 3$ mutant to the B6 standard strain are not rejected at this time. These results are consistent with the hypothesis that changes in the $\mathrm{CNBr}$ Ia fragment cause structural alterations in the histocompatibility antigens, which evoke a strong immunological response in the B6 standard strain. Changes that occur in the bm3-Ib $\mathrm{CNBr}$ fragment alter the structure of the histocompatibility antigens to a lesser extent and, therefore, evoke a poorer response in B6.

Some liver to kidney grafts from $b m 1$ to $b m 3$ and $b m 3$ to $b m 1$ survive at $70 \mathrm{~d}$ after grafting in spite of the structural differences in these mutants. These structural differences do cause acute rejection of skin grafts between these strains (Table 1).

It is generally accepted that MLC and CML are tests of cellular immune activity. Cellular immune activity is also the major potentiator of graft rejection. Therefore, a higher MLC stimulation index would be expected in donor-recipient combinations in which grafts are rejected. Rejection of grafts would also be expected when positive CML occurs. CML is positive in all combinations that we have tested (Table 1) but liver to kidney graft results vary. Although $b m 3-\mathrm{B} 6$ grafts survive longer than $b m 1$ $\mathrm{B} 6$ grafts, MLC stimulation index is higher in $b m 3-\mathrm{B} 6$ combinations then in $b m 1$ - $\mathrm{B} 6$ combinations (Table 1). CML and MLC among $\mathrm{H}-2^{\mathrm{b}}$ mutants and their parentals do not correlate with liver tissue graft results.

Male to female liver tissue grafts are rejected in the $b m 1\left(H-2 K^{b}\right)$ mutant by $56 \mathrm{~d}$ after grafting and in the $b m 3\left(H-2 K^{b}\right)$ mutant by $70 \mathrm{~d}$ after grafting. In the B6 parentals, some male to female grafts still survive at $105 \mathrm{~d}$ (Fig. 1). The mutational events in $b m 1$ and $b m 3$ appear to have altered either the immune response of the female to antigenic sites on male liver or the manner of presentation of those sites to the female immune system.

Mutant strains $d m 1$ and $b m 14$ are both $H-2 D$ mutants. The $H-2^{d}$ mutant, $d m l$, is similar to the $\mathrm{H}-2^{\mathrm{b}}$ mutant, $b m 14$, in that parental to mutant liver grafts are viable 
at $70 \mathrm{~d}$ after grafting and as long as $140 \mathrm{~d}$ in the case of B10.D2 to $d m 1$. However, the reciprocal combination in the $\mathrm{H}-2^{\mathrm{d}}$ mutant ( $d m 1$ to $\left.\mathrm{B} 10 . \mathrm{D} 2\right)$ is rejected by $35 \mathrm{~d}$ after grafting. This result would indicate a gain in an antigenic site or sites in the $\mathrm{dm} 1$ mutant critical in the liver grafting system. A reciprocal difference is noted between $d m 1$ and B10.D2 in MLC depending on which strain is employed as stimulator and which is employed as responder. B10.D2 stimulates $d m 1$ considerably more than $d m 1$ stimulates B10.D2 (Table 1). Since the opposite liver graft results from those observed would be predicted from MLC data, it would appear that liver graft reactions cannot be predicted by MLC results in this mutant-parental combination.

We previously reported (Schultz et al. 1977, 1981) that it is the $K$ rather than the $D$ region of the $\mathrm{MHC}$ that has major control over liver graft survival. The minor effect on this grafting system of the mutational changes in the fm2 and $\mathrm{bm} 14(\mathrm{H}-2 \mathrm{D})$ mutants as compared with effects of the mutations in $b m 1, b m 3$, and $b m 4(H-2 K)$ mutants support these findings (See Fig. 1).

Both bm1 and bm 3 grafts elicit greater immune response in $b m 14$ animals than do bm14 grafts in $b m 1$ and $b m 3$ mice. Thus $b m 14$ behaves much like the B6 parental strain in the liver graft system; further evidence that changes in the $H-2 D^{b}$ region fail to affect liver graft survival in the same way as they effect skin graft survival.

However, the $d m 1$ to B10.D2 graft results argue for the importance of the $D$ end of the MHC in control of liver tissue graft survival, since these grafts are rejected as early as $35 \mathrm{~d}$ after grafting. Approximately $30 \%(6-7)$ of the peptides of the H-2D molecule have been altered in the $d m 1$ mutant (Brown et al. 1978, Nairn 1980). In addition, there are changes in the carbohydrate side chains of the $H-2 \mathrm{D}^{\mathrm{dm} 1}$ molecule. The $H-2 L$ region of this mutant has also been altered (Bennett et al. 1980 , Wilson et al. 1982). Antigens are present in $d m 1$ that are absent in B10.D2 and the possibility exists that $\mathrm{D}^{\mathrm{dm} 1}$ is a hybrid molecule. The $d m 1$ mutant phenotype, therefore, is unexpectedly complex and very different from the B10.D2 phenotype. The extent of the biochemical changes in this mutant and/or the regions covered by these changes $(H-2 D$ and $H-2 L)$ may explain the vigorous rejection of $d m 1$ liver grafts by B10.D2. It must be noted, however, that, although these large differences exist between the structures of the $\mathrm{H}-2 \mathrm{D}^{\mathrm{d}} \mathrm{L}^{\mathrm{d}}$ and the $\mathrm{H}-2 \mathrm{D}^{\mathrm{dm} 1} \mathrm{~L}^{\mathrm{dm} 1}$ molecules, liver grafts from B10.D2 to $d m 1$ survive as long as $140 \mathrm{~d}$ after grafting. The immune response of females to grafts of male liver is not altered by these mutational changes since $d m 1$ male to female liver grafts do not differ in survival pattern from B10.D2 male to female grafts.

In summary, our data show that skin graft, CML, MLC, and serological reactivity cannot be used to predict rejection or survival of liver tissue. We can tentatively report that differences in the biochemical structure of $\mathrm{H}-2$ antigens correlate with liver tissue graft behavior. Alterations in the H-2D region have a limited effect on liver graft survival as compared with changes in the $H-2 \mathrm{~K}$ or $H-2 \mathrm{D}$ plus $H-2 L$ regions. Moreover, it appears that alterations in different peptide fragments of the $\mathrm{H}-2 \mathrm{~K}^{\mathrm{b}}$ antigen may differentially effect the ability of a liver graft to elicit an immune response. Several explanations are possible for the differences noted in liver and skin graft survival in $\mathrm{H}-2$ mutant strains. It is most likely that different $\mathrm{T}$-cell subsets are involved in these two responses. $\mathrm{T}$ cells that are responsible for liver tissue rejection may carry different receptors from $\mathrm{T}$ cells responsible for skin graft rejection. Additionally, these receptors may look at 
different sites on the $\mathrm{H}-2$ molecule or the $\mathrm{H}-2$ molecule may be presented differently on liver cells than on skin cells. Further studies will be necessary to enable us to establish the exact biological events which underlie differences in the host response to grafts of these two tissues.

Acknowledgments. This work was supported by the Medical Research Service of the Veterans Administration. The authors thank Mary Bouwsma for excellent technical assistance and Icey Heningburg and Joyce Farrar for typing of the manuscript. We also thank Dr. Rod Nairn for critical reading of and assistance in the preparation of this manuscript.

\section{References}

Apt, A. S., Blandova, Z. K., Dishkant, I. P., Shumova, T. E., Vedernikov, A. A., and Egorov, I. K.: Study of $\mathrm{H}-2$ mutations in mice: IV. A comparison of the mutants $\mathrm{M} 505$ and $\mathrm{Hzl}$ by skin grafting and serological techniques. Immunogenetics 1: 444-451, 1975

Bailey, D.W. and Usama, B.: A rapid method of skin grafting on tails of mice. Transplant. Bull. 7: 424 425,1960

Ben-Nun, A., Maron, R., Ron, Y., and Cohen, I. R.: H-2 gene products influence susceptibility of target thyroid gland to damage in experimental autoimmune thyroiditis. Eur. J. Immunol. 10: 156-159, 1980

Bennett, M., Melvold, R. W., and Kohn, H. I.: Bone-marrow-cell grafts involving the $H-2 D$ and $H-2 L$ mutant haplotypes. Immunogenetics 10: 273-281, 1980

Brown, J. L. and Nathenson, S.: Structural differences between parent and mutant H-2K glycoproteins from two $H-2 K$ gene mutants: $B 6 . C-H-2^{b a}(H z 1)$ and B6-H-2 $2^{b d}(M 505)$. J. Immunol. 118: 98-102, 1977

Brown, J. L., Nairn, R., and Nathenson, S. G.: Structural differences between the mouse $H-2 D$ products of the mutant B10.D2.M504 $\left(H-2^{d a}\right)$ and the parental nonmutant strain B10.D2 $\left(H-2^{d}\right)$.J.Immunol. 120 : $726-733,1978$

Davis, W.C., McKenzie, I. F.C., and Melvold, R.W.: A comparison of skin and heart graft reaction patterns in H-2 mutant mice. Transplantation 29: 189-192, 1980

DeMott-Friberg, R., Beals, T.F., and Schultz, J. S.: $H-2$ and background influences on tissue grafts across the H-Y barrier. Immunogenetics 9: 369-381, 1979

Egorov, I. K.: Genetic control of $H-2$ alloantigen as inferred from analysis of mutations. Immunogenetics 1: 97-107, 1974

Egorov, I. K., Pospelov, L. E., Shkurko, V. N., Mnatsakanyan, Y. A., and Khodjaen, E. Y.: Study of $\mathrm{H}-2$ mutations in mice. VIII. Aberrant expression of H-2.5 Antigen on Red Blood Cells of $\mathrm{H}-2^{b}$ mutants. Immunogenetics 8: 161-170, 1979

Ewenstein, B. M., Uehara, H., Nisizawa, T., Melvold, R. W., Kohn, H. I., and Nathenson, S. G.: Biochemical studies on the $\mathrm{H}-2 \mathrm{~K}$ antigens of the MHC mutants bm3 and bm11. Immunogenetics 11: 388-395, 1980

Klein, J., Chiang, C. L., Lofgreen, J., and Steinmuller, D.: Participation of $H$-2 regions in heart-transplant rejection. Transplantation 22: 384-390, 1976

Klein, J.: $H-2$ Mutations: Their genetics and effect on immune functions. Adv. Immunol. 26: 56-146, 1978

Kohn, H. I., Klein, J., Melvold, R. W., Nathenson, S. G., Pious, D., and Shreffler, D. C.: The first $\mathrm{H}-2$ mutant workshop. Immunogenetics 7: 279-294, 1978

McKenzie, I. F. C., Morgan, G. M., Sandrin, M. S., Michaelides, M. M., Melvold, R. W., and Kohn, H. I.: B6.C- $H-2^{b m 12}$, A new $H-2$ mutation in the I region in the mouse. J. Exp. Med. 150:1323-1338, 1979

Melief, C. J. M., de Waal, L. P., van der Meulen, M.Y., Melvold, R. W., and Kohn, H. I.: Fine specificity of alloimmune cytotoxic T lymphocytes directed against $H-2 K . J$. Exp. Med. 151: 993-1013, 1980

Nairn, R., Yamaga, K., and Nathenson, S. G.: Biochemistry of the gene products from murine MHC mutants. Annu. Rev. Genet. 14: 241-277, 1980

Nisizawa, T., Ewenstein, B. M., Uehara, H., McGovern, D., and Nathenson, S. G.: Biochemical Studies on the $\mathrm{H}-2 \mathrm{~K}$ antigens of the MHC mutant bm1. Immunogenetics 12: 33-44, 1981 
Schultz, J. S., Beals, T. F., and Petraitis, F. P.: Tissue graft rejection in mice. I. Contributions of $H-2$ and non- $\mathrm{H}-2$ genetic barriers. Immunogenetics 3: 85-96, 1976

Schultz, J. S., Beals, T.F., and DeMott-Friberg, R.: Tissue graft rejection in mice. V. Survival, rejection, or proliferation across minor barriers. Immunogenetics 6:585-595, 1978

Schultz, J. S., DeMott-Friberg, R., and Beals, T. F.: Immunogenetic control of the response of female mice to male tissue grafts. Immunogenetics 12: 199-205, 1981

Streilein, J. W. and Klein, J.: Neonatal tolerance induction across $H-2$ mutational disparity: Induction and specificity of tolerance: Immunogenetics $10: 113-123,1980$

Wilson, P. H., Nairn, R., Nathenson, S. G., and Sears, D. W.: Unexpected complexity of the $d m 1$ mutation revealed in the structures of three H-2D/L-related antigens: Immunogenetics 15: 225-237, 1982

$\mathrm{Wu}, \mathrm{S}$., Bach, F.H., and Sopori, M.: Differential alloresponse of murine thymocytes to $H-2 \mathrm{~K}$ region different recombinants and to $H-2 K^{b}$ mutants: J. Immunol. 124: 2464-2467, 1980

Received January 7, 1982 